\title{
Factorization of the Two Loop Four-Particle Amplitude in Superstring Theory Revisited
}

\author{
Zhi-Guang Xiao* \\ School of Physics, Peking University \\ Beijing 100871, P. R. China \\ Chuan-Jie $\mathrm{Zhu}^{\dagger \dagger}$ \\ Institute of Theoretical Physics, Chinese Academy of Sciences \\ P. O. Box 2735, Beijing 100080, P. R. China
}

October 22, 2018

\begin{abstract}
We study in detail the factorization of the newly obtained two-loop four-particle amplitude in superstring theory. In particular some missing factors from the scalar correlators are obtained correctly, in comparing with a previous study of the factorization in two-loop superstring theory. Some details for the calculation of the factorization of the kinematic factor are also presented.
\end{abstract}

\footnotetext{
*E-mail: xiaozhiguang@pku.edu.cn

$\dagger$ E-mail: zhucj@itp.ac.cn

¥Supported in part by fund from the National Natural Science Foundation of China with grant Number 90103004 and 10475104.
} 
Explicit result for higher loop amplitudes in superstring is quite rare. To our knowledge the only explicitly known higher loop $(\geq 2)$ non-vanishing amplitude is the four-particle amplitude in superstring theory, firstly obtained in 1 and later re-obtained in 2, 3] in an explicitly gauge independent way, following the works of D' Hoker and Phong 4, 5, 6, 6, 8, on two loop measure of superstring theory. Recently D' Hoker and Phong 90 10] also gave a measure for three loop superstring theory. It remains to see if this can be used to do explicit three loop computations in superstring theory. For other promising approach of covariant calculation of superstring amplitudes we refer the reader to Berkovits's review [11.

Due to the rareness of explicit results, it is natural to study the known result in depth. The old result was casted into an explicit modular invariant form [12 and used in [13] to prove the vanishing of the $R^{4}$ correction 14 15. It has also been proved in [16] that the results obtained in [1, 2 3] are equivalent. Another goal we have in mind is to make connection with known results from field theory in $\mathcal{N}=4$ supersymmetric Yang-Mills theory 17 18. This seems a trivial problem, but in fact it turns out to be quite tricky. One could use unitarity to fix the overall factor for the fourparticle amplitude. In order to do this we need to know the precise overall factor for other one loop amplitudes involved. Due to the incomplete results in the literature, we therefore computed all the relevant amplitudes in a consistent way and fixed all the overall factors by either using factorization or unitarity. We will present a detailed study of factorization and unitarity in superstring theory in a later publication [19. In this paper we present only the result of factorization of the two loop four-particle amplitude in superstring theory.

The factorization of the two loop four-particle amplitude in superstring theory was studied before in 20] 21. Compared with these studies, we improved on obtaining some missing factors from the scalar correlators. Also some details for the calculation of the factorization of the kinematic factor are presented. We will need these results in the forthcoming paper [19.

To begin with, let us recall the two-loop four-particle amplitude in type II superstring theories obtained in refs. [2, 3]:

$$
\begin{aligned}
\mathcal{A}_{I I}= & C_{I I}(2 \pi)^{10} \delta^{10}\left(\sum_{i} k_{i}\right) g_{c}^{4} K\left(k_{i}, \epsilon_{i}\right) \int \frac{1}{T^{5}} \frac{\prod_{i=1}^{6} \mathrm{~d}^{2} a_{i}}{\mathrm{~d} V_{p r}\left|\prod_{i<j} a_{i j}\right|^{2}} \\
& \times \prod_{i=1}^{4} \frac{\mathrm{d}^{2} z_{i}}{\left|y\left(z_{i}\right)\right|^{2}} \prod_{i<j} \exp \left\{-k_{i} \cdot k_{j}\left\langle X\left(z_{i}\right) X\left(z_{j}\right)\right\rangle\right\}, \\
& \times\left|s\left(z_{1} z_{2}+z_{3} z_{4}\right)+t\left(z_{1} z_{4}+z_{2} z_{3}\right)+u\left(z_{1} z_{3}+z_{2} z_{4}\right)\right|^{2},
\end{aligned}
$$

where

$$
\begin{aligned}
\mathrm{d} V_{p r} & =\frac{\mathrm{d}^{2} a_{i} \mathrm{~d}^{2} a_{j} \mathrm{~d}^{2} a_{k}}{\left|a_{i j} a_{i k} a_{j k}\right|^{2}}, \\
T & =\int \mathrm{d}^{2} z_{1} \mathrm{~d}^{2} z_{2} \frac{\left|z_{1}-z_{2}\right|^{2}}{\left|y\left(z_{1}\right) y\left(z_{2}\right)\right|^{2}},
\end{aligned}
$$




$$
y^{2}(z)=\prod_{i=1}^{6}\left(z-a_{i}\right),
$$

and $\left\langle X\left(z_{i}\right) X\left(z_{j}\right)\right\rangle \equiv\left\langle X\left(z_{i}, \bar{z}_{i}\right) X\left(z_{j}, \bar{z}_{j}\right)\right\rangle$ 's are the scalar correlators (see below in eq. (19) for explicit formulas in terms of prime form and holomorphic differentials). The $K\left(k_{i}, \epsilon_{i}\right)$ is the standard kinematic factor appearing at tree, one- and two-loop computations [22, 1, 3]. $C_{I I}$ is an overall factor which should be determined from factorization and unitarity [19].

In the dividing degeneration limit: $a_{2}-a_{1}=u, a_{3}-a_{1}=v u, u \rightarrow 0$, we have

$$
T \rightarrow \frac{2}{|u|} T_{1} T_{2} \frac{1}{\left|a_{14} a_{15} a_{16}\right|}
$$

where

$$
\begin{aligned}
T_{i} & =\int \frac{\mathrm{d}^{2} z}{\left|y_{i}(z)\right|^{2}}, \\
y_{1}(z) & =\sqrt{z(z-1)(z-v)}, \\
y_{2}(z) & =\sqrt{\left(z-a_{1}\right)\left(z-a_{4}\right)\left(z-a_{5}\right)\left(z-a_{6}\right)} .
\end{aligned}
$$

Setting

$$
z_{1}=x_{1} u+a_{1}, \quad \text { and } \quad z_{2}=x_{2} u+a_{1},
$$

we have:

$$
\begin{aligned}
& \frac{\left|\prod_{i<j} a_{i j}\right|^{2}}{\left|a_{45} a_{46} a_{56}\right|^{2}} \rightarrow|u|^{6}|v(1-v)|^{2}\left|a_{14} a_{15} a_{16}\right|^{6}, \\
&\left|y\left(z_{1}\right)\right|^{2} \rightarrow|u|^{3}\left|y_{1}\left(x_{1}\right)\right|^{2}\left|a_{14} a_{15} a_{16}\right|, \\
&\left|y\left(z_{4}\right)\right|^{2} \rightarrow\left|\left(z_{4}-a_{1}\right)\right|^{2}\left|y_{2}\left(z_{4}\right)\right|^{2}, \\
& \prod_{i=1}^{4}\left|y\left(z_{i}\right)\right|^{2} \rightarrow|u|^{6}\left|a_{14} a_{15} a_{16}\right|^{2}\left|\left(z_{3}-a_{1}\right)\left(z_{4}-a_{1}\right)\right|^{2} \\
& \mathrm{~d}^{2} a_{1} \mathrm{~d}^{2} a_{2} \mathrm{~d}^{2} a_{3} \rightarrow\left|y_{1}\left(x_{1}\right) y_{1}\left(x_{2}\right) y_{2}\left(z_{3}\right) y_{2}\left(z_{4}\right)\right|^{2}, \\
& \mathrm{~d}^{2} z_{1} \mathrm{~d}^{2} z_{2} \rightarrow|u|^{2} \mathrm{~d}^{2} u \mathrm{~d}^{2} v \mathrm{~d}^{2} a_{1}, \\
& \mid s\left(z_{1} z_{2}+z_{3} z_{4}\right)+ t\left(z_{1} z_{4}+z^{2} x^{2} x_{1} \mathrm{~d}^{2} x_{2},\right. \\
& \rightarrow\left.|s|^{2} \mid\left(z_{3}-a_{1}\right)\left(z_{4}-a_{1}\right) z_{3} . z_{2} z_{4}\right)\left.\right|^{2} .
\end{aligned}
$$

By using the above results we have

$$
\begin{aligned}
\mathcal{A}_{I I} \rightarrow & C_{I I}(2 \pi)^{10} \delta^{10}\left(\sum_{i} k_{i}\right) g_{c}^{4} K\left(k_{i}, \epsilon_{i}\right) \\
& \times \int \frac{\mathrm{d}^{2} u}{|u| 2^{5} T_{1}^{5} T_{2}^{5}} \frac{\mathrm{d}^{2} a_{1}}{\left|a_{14} a_{15} a_{16}\right|^{3}} \frac{\mathrm{d}^{2} v}{|v(v-1)|^{2}} \\
& \times \frac{\mathrm{d}^{2} x_{1} \mathrm{~d}^{2} x_{2}}{\left|y_{1}\left(x_{1}\right) y_{1}\left(x_{2}\right)\right|^{2}} \frac{\mathrm{d}^{2} z_{3} \mathrm{~d}^{2} z_{4}}{\left|y_{2}\left(z_{3}\right) y_{2}\left(z_{4}\right)\right|^{2}} \\
& \times s^{2} \prod_{i<j} \exp \left\{-k_{i} \cdot k_{j}\left\langle X\left(z_{i}\right) X\left(z_{j}\right)\right\rangle\right\} .
\end{aligned}
$$




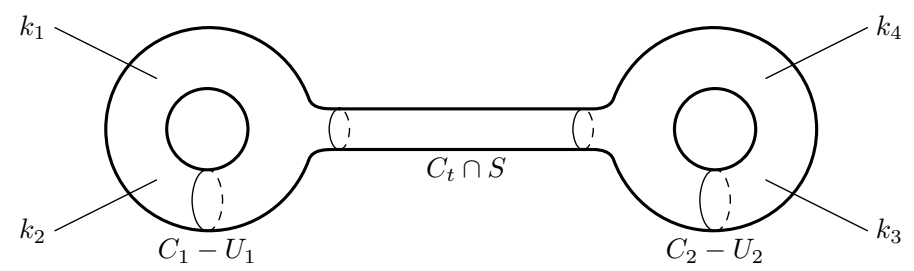

Figure 1: The dividing degeneration limit of a genus 2 surface.

In order to prove that the above amplitude factorizes correctly, we need to study the degeneration limit of the scalar correlators.

The expression in the exponential factor in eq. (17) is:

$$
\begin{aligned}
-\sum_{i<j} k_{i} \cdot k_{j}\left\langle X\left(z_{i}\right) X\left(z_{j}\right)\right\rangle= & \frac{s}{2}\left(\left\langle X\left(z_{1}\right) X\left(z_{2}\right)\right\rangle+\left\langle X\left(z_{3}\right) X\left(z_{4}\right)\right\rangle\right. \\
& \left.-\left\langle X\left(z_{1}\right) X\left(z_{3}\right)\right\rangle-\left\langle X\left(z_{2}\right) X\left(z_{4}\right)\right\rangle\right) \\
& +\frac{t}{2}\left(\left\langle X\left(z_{1}\right) X\left(z_{4}\right)\right\rangle+\left\langle X\left(z_{2}\right) X\left(z_{3}\right)\right\rangle\right. \\
& \left.-\left\langle X\left(z_{1}\right) X\left(z_{3}\right)\right\rangle-\left\langle X\left(z_{2}\right) X\left(z_{4}\right)\right\rangle\right) .
\end{aligned}
$$

where the scalar correlator $\left\langle X\left(z_{i}\right) X\left(z_{j}\right)\right\rangle$ is given as follows:

$$
\begin{aligned}
\left\langle X\left(z_{i}\right) X\left(z_{j}\right)\right\rangle= & -\ln \left(\left|E\left(z_{i}, z_{j}\right)\right|^{2}\right) \\
& +2 \pi\left(\operatorname{Im} \int_{z_{i}}^{z_{j}} \omega\right)(\operatorname{Im} \tau)^{-1}\left(\operatorname{Im} \int_{z_{i}}^{z_{j}} \omega\right),
\end{aligned}
$$

in terms of the prime form $E\left(z_{i}, z_{j}\right)$, the period matrix:

$$
\tau=\left(\begin{array}{ll}
\tau_{11} & \tau_{12} \\
\tau_{21} & \tau_{22}
\end{array}\right)
$$

and the holomorphic differentials:

$$
\omega(z)=\left(\begin{array}{l}
\omega_{1}(z) \\
\omega_{2}(z)
\end{array}\right)
$$

In the degeneration limit $u=t^{4} \rightarrow 0$ (see Fig. 1), we have the following explicit formulas from Fay's book [23] (page 38, Proposition 3.1 and page 41, Corollary 3.2):

$$
\begin{aligned}
& \omega_{1}(x, t)= \begin{cases}v_{1}(x)+\frac{t}{4} v_{1}\left(p_{1}\right) u_{1}\left(x, p_{1}\right)+O\left(t^{2}\right), & \left(x \in C_{1}-U_{1}\right) \\
\frac{t}{4} v_{1}\left(p_{1}\right) u_{2}\left(x, p_{2}\right)+O\left(t^{2}\right), & \left(x \in C_{2}-U_{2}\right)\end{cases} \\
& \omega_{2}(x, t)= \begin{cases}\frac{t}{4} v_{2}\left(p_{2}\right) u_{1}\left(x, p_{1}\right)+O\left(t^{2}\right), & \left(x \in C_{1}-U_{1}\right) \\
v_{2}(x)+\frac{t}{4} v_{2}\left(p_{2}\right) u_{2}\left(x, p_{2}\right)+O\left(t^{2}\right), & \left(x \in C_{2}-U_{2}\right)\end{cases}
\end{aligned}
$$

and

$$
\tau \rightarrow\left(\begin{array}{cc}
\tau_{1} & 0 \\
0 & \tau_{2}
\end{array}\right)+2 \pi i \frac{t}{4}\left(\begin{array}{cc}
v_{1}^{2}\left(p_{1}\right) & v_{1}\left(p_{1}\right) v_{2}\left(p_{2}\right) \\
v_{1}\left(p_{1}\right) v_{2}\left(p_{2}\right) & v_{2}^{2}\left(p_{2}\right)
\end{array}\right)+O\left(t^{2}\right)
$$


Here $v_{i}(z)$ is the holomorphic differential on the $i$-th torus (see below in eq. (30) for explicit expression). $p_{1}$ is the $\infty$-point on the first torus $\left(p_{1}=\infty\right)$ and $p_{2}$ is one of the branch point on the second torus $\left(p_{2}=a_{1}\right)$. The Abelian differential of the second kind $u_{i}$ on the two tori are given as follows:

$$
\begin{aligned}
u_{i}(x, y) & =-v_{i}(x) v_{i}(y) \frac{\partial^{2} \ln \vartheta}{\partial^{2} z}\left(e_{i}\right), \\
e_{i} & =\int_{x}^{y} v_{i}-\frac{1}{2}\left(\tau_{i}+1\right) .
\end{aligned}
$$

By using the above results and the degeneration property of the theta function, the correlators are degenerated as follows:

$$
\begin{aligned}
& \left\langle X\left(z_{1}\right) X\left(z_{2}\right)\right\rangle \rightarrow-G_{1}\left(x_{1}, x_{2}\right)+\ln \left|v_{1}\left(x_{1}\right) v_{1}\left(x_{2}\right)\right|-\ln |u|^{2}, \\
& \left\langle X\left(z_{3}\right) X\left(z_{4}\right)\right\rangle \rightarrow-G_{2}\left(z_{3}, z_{4}\right)+\ln \left|v_{2}\left(z_{3}\right) v_{2}\left(z_{4}\right)\right|, \\
& \left\langle X\left(z_{1}\right) X\left(z_{3}\right)\right\rangle \rightarrow-G_{1}\left(x_{1}, p_{1}\right)-G_{2}\left(p_{2}, z_{3}\right)+\ln \left|v_{1}\left(x_{1}\right) v_{2}\left(z_{3}\right)\right| \\
& -\ln \left|K_{1} K_{2} / 4\right|-\ln |u|^{1 / 2}-\ln \left|a_{14} a_{15} a_{16}\right|^{1 / 2},(27) \\
& \left\langle X\left(z_{2}\right) X\left(z_{4}\right)\right\rangle \quad \rightarrow \quad-G_{1}\left(x_{2}, p_{1}\right)-G_{2}\left(p_{2}, z_{4}\right)+\ln \left|v_{1}\left(x_{2}\right) v_{2}\left(z_{4}\right)\right| \\
& -\ln \left|K_{1} K_{2} / 4\right|-\ln |u|^{1 / 2}-\ln \left|a_{14} a_{15} a_{16}\right|^{1 / 2},
\end{aligned}
$$

where we have defined:

$$
G_{i}(x, y)=\ln \left|\frac{\vartheta_{1}\left(\int_{x}^{y} v_{i}, \tau_{i}\right)}{\partial \vartheta_{1}\left(0, \tau_{i}\right)}\right|^{2}-2 \pi\left(\operatorname{Im} \int_{x}^{y} v_{i}\right)\left(\operatorname{Im} \tau_{i}\right)^{-1}\left(\operatorname{Im} \int_{x}^{y} v_{i}\right),
$$

and

$$
\begin{aligned}
v_{1}(z) & =\frac{\mathrm{d} x}{K_{1} y_{1}(x)}=\mathrm{d} \tilde{x}, \quad v_{2}(z)=\frac{\mathrm{d} z}{K_{2} y_{2}(z)}=\mathrm{d} \tilde{z} \\
K_{1} & =\oint_{\alpha_{1}} \frac{\mathrm{d} x}{y_{1}(x)}, \quad K_{2}=\oint_{\alpha_{2}} \frac{\mathrm{d} z}{y_{2}(z)}
\end{aligned}
$$

By using these results we have:

$$
\begin{aligned}
-\sum_{i<j} k_{i} \cdot k_{j}\left\langle X\left(z_{i}\right) X\left(z_{j}\right)\right\rangle \\
\rightarrow \quad \frac{s}{2}\left[-G_{1}\left(x_{1}, x_{2}\right)-G_{2}\left(z_{3}, z_{4}\right)+G_{1}\left(x_{1}, p_{1}\right)\right. \\
+G_{2}\left(p_{2}, z_{3}\right)+G_{1}\left(x_{2}, p_{1}\right)+G_{2}\left(p_{2}, z_{4}\right) \\
\left.+\ln \left(\left|a_{14} a_{15} a_{16}\right|\left|K_{1} K_{2} / 4\right|^{2}\right)\right]+\ln |u|^{-s / 2}
\end{aligned}
$$

and so the two loop four-boson amplitude becomes:

$$
\begin{aligned}
\mathcal{A}_{I I} \rightarrow & i C_{I I}(2 \pi)^{10} \delta^{10}\left(\sum_{i} k_{i}\right) g_{c}^{4} K\left(k_{i}, \epsilon_{i}\right) \\
& \int \frac{\mathrm{d}^{2} u}{2^{5} T_{1}^{5} T_{2}^{5}} \frac{\mathrm{d}^{2} a_{1}}{\left|a_{14} a_{15} a_{16}\right|^{3}} \frac{\mathrm{d}^{2} v}{|v(v-1)|^{2}} \\
& \times \frac{\mathrm{d}^{2} x_{1} \mathrm{~d}^{2} x_{2}}{\left|y_{1}\left(x_{1}\right) y_{1}\left(x_{2}\right)\right|^{2}} \frac{\mathrm{d}^{2} z_{3} \mathrm{~d}^{2} z_{4}}{\left|y_{2}\left(z_{3}\right) y_{2}\left(z_{4}\right)\right|^{2}}
\end{aligned}
$$




$$
\begin{aligned}
& \times \exp \left\{\frac { s } { 2 } \left(-G_{1}\left(x_{1}, x_{2}\right)-G_{2}\left(z_{3}, z_{4}\right)+G_{1}\left(x_{1}, p_{1}\right)\right.\right. \\
& \left.\left.\quad+G_{2}\left(p_{2}, z_{3}\right)+G_{1}\left(x_{2}, p_{1}\right)+G_{2}\left(p_{2}, z_{4}\right)\right)\right\} \\
& \times s^{2}\left|a_{14} a_{15} a_{16}\right|^{s / 2}\left|K_{1} K_{2} / 4\right|^{s}|u|^{-s / 2-1} \\
& \rightarrow \quad i C_{I I}(2 \pi)^{10} \delta^{10}\left(\sum_{i} k_{i}\right) g_{c}^{4} K\left(k_{i}, \epsilon_{i}\right) \\
& \times \frac{16 \pi}{-s+2} \int \frac{1}{2^{5} T_{1}^{5} T_{2}^{5}} \frac{\mathrm{d}^{2} a_{1}}{\left|a_{14} a_{15} a_{16}\right|^{2}} \frac{\mathrm{d}^{2} v}{|v(v-1)|^{2}} \\
& \times \frac{\mathrm{d}^{2} x_{1} \mathrm{~d}^{2} x_{2}}{\left|y_{1}\left(x_{1}\right) y_{1}\left(x_{2}\right)\right|^{2}} \frac{\mathrm{d}^{2} z_{3} \mathrm{~d}^{2} z_{4}}{\left|y_{2}\left(z_{3}\right) y_{2}\left(z_{4}\right)\right|^{2}} \times\left|K_{1} K_{2} / 4\right|^{2} \\
& \times \exp \left\{-\left(G_{1}\left(x_{1}, x_{2}\right)-G_{1}\left(x_{1}, p_{1}\right)-G_{1}\left(x_{2}, p_{1}\right)\right)\right. \\
& \left.\quad-\left(G_{2}\left(z_{3}, z_{4}\right)-G_{2}\left(z_{3}, p_{2}\right)-G_{2}\left(z_{4}, p_{2}\right)\right)\right\} .
\end{aligned}
$$

In order to prove that the above amplitude do factorize correctly, we need to do two things. The first is to prove that the kinematic factor $K\left(k_{i}, \epsilon_{i}\right)$ factorizes into a summation of two one-loop 3-particle kinematic factor. The summation is over all possible intermediate states. The second is to prove that the rests in eq. (33) are the product of two one-loop 3particle amplitudes apart from the propagator $\frac{1}{-s+2}$. Let us study the second first.

The first non-vanishing contributions is from a massive intermediate states. Only the massive boson from the NS-NS sector contributes because of the super-ghost. So we need to compute the 1-loop amplitude for two massless bosons to one massive boson. The massive boson vertex operator in the 0-picture 24 25] 26] is:

$$
\begin{aligned}
\mathcal{V}_{M}^{(0,0)}( & \left.k, \alpha_{\mu \nu \rho}, \sigma_{\mu \nu}, \sigma_{\mu}\right) \\
= & -g_{M}\left\{\alpha_{[\mu \nu \rho]}\left[3 i \partial X^{\mu} \psi^{\nu} \psi^{\rho}-k \cdot \psi \psi^{\mu} \psi^{\nu} \psi^{\rho}\right]\right. \\
& +\sigma_{\mu \nu}\left[i \partial X^{\mu} \partial X^{\nu}+\partial \psi^{\mu} \psi^{\nu}+k \cdot \psi i \partial X^{\mu} \psi^{\nu}\right] \\
& \left.+\sigma_{\mu}\left[i \partial^{2} X+k \cdot \psi \partial \psi^{\mu}\right]\right\}\{\text { right-moving part }\} e^{i k \cdot X},
\end{aligned}
$$

where $\alpha_{\mu \nu \rho}, \sigma_{\mu \nu}$ and $\sigma_{\mu}$ are the polarization tensors and we set the following normalization:

$$
\alpha_{\mu \nu \rho}(k) \alpha^{\mu \nu \rho}(-k)=-\frac{1}{6}, \quad \sigma_{\mu \nu}(k) \sigma^{\mu \nu}(-k)=1 .
$$

By convention $\alpha(-k)=\alpha^{*}(k)$ and $\sigma(-k)=\sigma^{*}(k)$. For later use we give here also the completeness relations for these tensors:

$$
\begin{aligned}
\sum_{\alpha} \alpha_{\mu_{1} \nu_{1} \rho_{1}}(k) \alpha_{\mu_{2} \nu_{2} \rho_{2}}(-k)= & -\frac{1}{288}\left(\left[\left(k_{\mu_{1}} k_{\mu_{2}}+2 g^{\mu_{1} \mu_{2}}\right)\right.\right. \\
& \times\left(k_{\nu_{1}} k_{\nu_{2}}+2 g^{\nu_{1} \nu_{2}}\right)\left(k_{\rho_{1}} k_{\rho_{2}}+2 g^{\rho_{1} \rho_{2}}\right) \\
-\left(\mu_{1} \leftrightarrow \nu_{1}\right) & \left.\left.-\left(\mu_{1} \leftrightarrow \rho_{1}\right)\right]-\left(\nu_{1} \leftrightarrow \rho_{1}\right)\right)
\end{aligned}
$$




$$
\begin{aligned}
& \sum_{\sigma} \sigma^{\mu_{1} \mu_{2}}(k) \sigma^{\nu_{1} \nu_{2}}(-k)=\frac{1}{8}\left[\left(k^{\mu_{1}} k^{\nu_{1}}+2 \eta^{\mu_{1} \nu_{1}}\right)\left(k^{\mu_{2}} k^{\nu_{2}}+2 \eta^{\mu_{2} \nu_{2}}\right)\right. \\
&\left.-\frac{1}{9}\left(k^{\mu_{1}} k^{\mu_{2}}+2 \eta^{\mu_{1} \mu_{2}}\right)\left(k^{\nu_{1}} k^{\nu_{2}}+2 \eta^{\nu_{1} \nu_{2}}\right)+\left(\mu_{1} \leftrightarrow \mu_{2}\right)\right] .
\end{aligned}
$$

The (bosonic) $n$-particle one loop amplitude are obtained as follows:

$$
\mathcal{A}_{n}^{1-\text { loop }}=\int \mathrm{d} \mu \prod_{i=1}^{n} \mathrm{~d}^{2} z_{i} \sum_{s} \eta_{s} Q_{s}\left\langle\prod_{i=1}^{n} \mathcal{V}\left(k_{i}, \epsilon_{i}, z_{i}\right)\right\rangle_{s},
$$

and for $n=3$ (two massless $\left(k_{1}\right.$ and $\left.k_{2}\right)$ and one massive $\left(k_{3}=k\right)$ ), we have

$$
\begin{aligned}
\mathcal{A}_{3}^{1-\text { loop }} & \\
= & -i g_{c}^{2} g_{M} C_{I}(2 \pi)^{10} \delta^{10}\left(k_{1}+k_{2}+k\right) K_{3} \\
& \times \int \mathrm{d} \mu \mathrm{d}^{2} z_{1} \mathrm{~d}^{2} z_{2} \mathrm{~d}^{2} z_{3} \frac{\prod_{i<j}\left|a_{i j}\right|^{2}}{64\left|y\left(z_{1}\right) y\left(z_{2}\right) y^{2}\left(z_{3}\right)\right|^{2}} \\
& \times \exp \left\{-\sum_{i<j} k_{i} \cdot k_{j}\left\langle X\left(z_{i}\right) X\left(z_{j}\right)\right\rangle\right\} \\
= & -i g_{c}^{2} g_{M} C_{I}(2 \pi)^{10} \delta^{10}\left(k_{1}+k_{2}+k\right) K_{3} \\
& \times \int \frac{1}{T^{6}} \frac{\prod_{i=1}^{4} \mathrm{~d}^{2} a_{i}}{\mathrm{~d} V_{p r}\left|\prod_{i<j} a_{i j}\right|^{2}} \frac{\mathrm{d}^{2} z_{1} \mathrm{~d}^{2} z_{2} \mathrm{~d}^{2} z_{3}}{64\left|y\left(z_{1}\right) y\left(z_{2}\right) y^{2}\left(z_{3}\right)\right|^{2}} \\
& \times \exp \left\{\left\langle X\left(z_{1}\right) X\left(z_{2}\right)\right\rangle-\left\langle X\left(z_{1}\right) X\left(z_{3}\right)\right\rangle-\left\langle X\left(z_{2}\right) X\left(z_{3}\right)\right\rangle\right\},
\end{aligned}
$$

where we have used the relation $2 k_{i} \cdot k_{j}=\left(k_{i}+k_{j}\right)^{2}-k_{i}^{2}-k_{j}^{2}$ to set $k_{1} \cdot k_{2}=-k_{1} \cdot k=-k_{2} \cdot k=-1$ and the kinematic factor $K_{3}$ is given as follows (for the left-part only):

$$
K_{3 L}=6 \alpha_{\mu \nu \rho} \epsilon_{2}^{\mu} \epsilon_{1}^{\nu} k_{2}^{\rho}+\sigma_{\mu \nu}\left(k_{1}^{\mu} k_{2}^{\nu} \epsilon_{1} \cdot \epsilon_{2}-k_{1}^{\mu} \epsilon_{2}^{\nu} \epsilon_{1} \cdot k_{2}-\epsilon_{1}^{\mu} \epsilon_{2}^{\nu}-\epsilon_{1}^{\mu} k_{2}^{\nu} k_{1} \cdot \epsilon_{2}\right) .
$$

By comparing the one-loop amplitude given in eq. (39) with the relevant expression in eq. (33), we see that there is a mismatch of one factor of $T_{i}$ and an extra integration over insertion point of the vertex operators. This is due to the fact that there is a translation invariance for the one-loop amplitude. So we can fix an insertion point and this cancels a factor $T$. The precise relation between $G$ and $\left\langle X\left(z_{i}\right) X\left(z_{j}\right)\right\rangle$ is:

$$
\left\langle X\left(z_{i}\right) X\left(z_{j}\right)\right\rangle=-G\left(z_{i}, z_{j}\right)-\ln \left|y\left(z_{i}\right) y\left(z_{j}\right) K^{2}\right| .
$$

(See also eqs. 25)-28) .) Taking this into account, we have

$$
\begin{aligned}
\int \frac{1}{T^{6}} & \frac{\mathrm{d}^{2} z_{1} \mathrm{~d}^{2} z_{2} \mathrm{~d}^{2} z}{\left|y\left(z_{1}\right) y\left(z_{2}\right) y^{2}(z)\right|^{2}} \\
& \times \exp \left\{\left\langle X\left(z_{1}\right) X\left(z_{2}\right)\right\rangle-\left\langle X\left(z_{1}\right) X(z)\right\rangle-\left\langle X\left(z_{2}\right) X(z)\right\rangle\right\} \\
= & \int \frac{|K|^{2}}{T^{6}} \frac{\mathrm{d}^{2} z_{1} \mathrm{~d}^{2} z_{2} \mathrm{~d}^{2} z}{\left|y\left(z_{1}\right) y\left(z_{2}\right) y(z)\right|^{2}} \\
& \times \exp \left\{-G\left(z_{1}, z_{2}\right)+G\left(z_{1}, z\right)+G\left(z_{2}, z\right)\right\} \\
= & \int \frac{|K|^{8}}{T^{6}} \mathrm{~d}^{2} \tilde{z}_{1} \mathrm{~d}^{2} \tilde{z}_{2} \mathrm{~d}^{2} \tilde{z} \exp \left\{-G\left(z_{1}, z_{2}\right)+G\left(z_{1}, z\right)+G\left(z_{2}, z\right)\right\}
\end{aligned}
$$




$$
\begin{aligned}
= & \int \frac{|K|^{6}}{T^{5}} \mathrm{~d}^{2} \tilde{z}_{1} \mathrm{~d}^{2} \tilde{z}_{2} \exp \left\{-G\left(z_{1}, z_{2}\right)+G\left(z_{1}, z\right)+G\left(z_{2}, z\right)\right\} \\
= & \int \frac{|K|^{2}}{T^{5}} \frac{\mathrm{d}^{2} z_{1} \mathrm{~d}^{2} z_{2}}{\left|y\left(z_{1}\right) y\left(z_{2}\right)\right|^{2}} \\
& \times \exp \left\{-G\left(z_{1}, z_{2}\right)+G\left(z_{1}, z\right)+G\left(z_{2}, z\right)\right\} .
\end{aligned}
$$

This agrees with eq. (33) for the relevant part of the two one-loop amplitudes.

To complete the study of the factorization we would also like to show that the kinematic factor $K\left(k_{i}, \epsilon_{i}\right)$ also factorizes correctly. This was done in [21, 20, 27]. Here we will give more details for the summation over intermediate tensors. This factorization is true for both the left- and right-part. Setting $\epsilon_{i}^{\mu \nu}=\epsilon_{i}^{\mu} \tilde{\epsilon}_{i}^{\nu}$, we have

$$
K\left(k_{i}, \epsilon_{i}\right)=K_{L}\left(k_{i}, \epsilon_{i}\right) K_{R}\left(k_{i}, \tilde{\epsilon}_{i}\right) .
$$

In the following we discuss the factorization of $K_{L}$ only and by a abuse of notation we simply write $K\left(k_{i}, \epsilon_{i}\right)$ for $K_{L}\left(k_{i}, \epsilon_{i}\right)$.

Setting

$$
\begin{aligned}
K_{3,1}\left(k_{1}, \epsilon_{1}, k_{2}, \epsilon_{2}, k, \alpha\right)= & 6 \epsilon_{1 \mu} \epsilon_{2 \nu} k_{1 \rho} \alpha^{\mu \nu \rho} \\
K_{3,2}\left(k_{1}, \epsilon_{1}, k_{2}, \epsilon_{2}, k_{3}, \sigma\right)= & -\sigma^{\mu \nu}\left(\epsilon_{1 \mu} \epsilon_{2 \nu}+k_{1 \mu} \epsilon_{2 \nu} \epsilon_{1} \cdot k_{2}\right. \\
& \left.+k_{1 \mu} k_{1 \nu} \epsilon_{1} \cdot \epsilon_{2}-\epsilon_{1 \nu} k_{1 \mu} \epsilon_{2} \cdot k_{1}\right),
\end{aligned}
$$

and by using the completeness relations eqs. (36) and (37) for the polarization tensors, we have

$$
\begin{aligned}
\sum_{\alpha} K_{3,1} & \left(k_{1}, \epsilon_{1}, k_{2}, \epsilon_{2}, k, \alpha\right) K_{3,1}\left(k_{3}, \epsilon_{3}, k_{4}, \epsilon_{4},-k, \alpha\right) \\
= & -\frac{1}{2}\left\{\left(u \left(\epsilon_{1} \cdot \epsilon_{4} \epsilon_{2} \cdot \epsilon_{3}-\epsilon_{1} \cdot \epsilon_{3} \epsilon_{2} \cdot \epsilon_{4}-\frac{1}{2} \epsilon_{2} \cdot \epsilon_{3} \epsilon_{1} \cdot k_{2} \epsilon_{4} \cdot k_{3}\right.\right.\right. \\
& +\frac{1}{2} \epsilon_{2} \cdot \epsilon_{4} \epsilon_{1} \cdot k_{2} \epsilon_{3} \cdot k_{4}-\frac{1}{2} \epsilon_{1} \cdot \epsilon_{4} \epsilon_{2} \cdot k_{1} \epsilon_{3} \cdot k_{4} \\
& \left.\left.+\frac{1}{2} \epsilon_{1} \cdot \epsilon_{3} \epsilon_{2} \cdot k_{1} \epsilon_{4} \cdot k_{3}\right)\right)+\epsilon_{1} \cdot \epsilon_{4} \epsilon_{2} \cdot \epsilon_{3}-\epsilon_{1} \cdot \epsilon_{3} \epsilon_{2} \cdot \epsilon_{4} \\
& +\epsilon_{2} \cdot \epsilon_{3}\left(-\epsilon_{1} \cdot k_{4} \epsilon_{4} \cdot k_{1}-\epsilon_{1} \cdot k_{3} \epsilon_{4} \cdot k_{2}\right) \\
& -\epsilon_{2} \cdot \epsilon_{4}\left(-\epsilon_{1} \cdot k_{4} \epsilon_{3} \cdot k_{1}-\epsilon_{1} \cdot k_{3} \epsilon_{3} \cdot k_{2}\right) \\
& +\epsilon_{1} \cdot \epsilon_{4}\left(-\epsilon_{2} \cdot k_{4} \epsilon_{3} \cdot k_{1}-\epsilon_{2} \cdot k_{3} \epsilon_{3} \cdot k_{2}\right) \\
& -\epsilon_{1} \cdot \epsilon_{3}\left(-\epsilon_{2} \cdot k_{4} \epsilon_{4} \cdot k_{1}-\epsilon_{2} \cdot k_{3} \epsilon_{4} \cdot k_{2}\right) \\
& +\left(\epsilon_{1} \cdot k_{4} \epsilon_{2} \cdot k_{3}-\epsilon_{1} \cdot k_{3} \epsilon_{2} \cdot k_{4}\right) \\
& \left.\times\left(\epsilon_{3} \cdot k_{2} \epsilon_{4} \cdot k_{1}-\epsilon_{3} \cdot k_{1} \epsilon_{4} \cdot k_{2}\right)\right\}
\end{aligned}
$$

and

$$
\begin{aligned}
\sum_{\sigma} & K_{3,2}\left(k_{1}, \epsilon_{1}, k_{2}, \epsilon_{2}, k, \sigma\right) K_{3,2}\left(k_{3}, \epsilon_{3}, k_{4}, \epsilon_{4},-k, \sigma\right) \\
= & \frac{1}{2}\left\{\frac{1}{2} u^{2} \epsilon_{1} \cdot \epsilon_{2} \epsilon_{3} \cdot \epsilon_{4}+u\left[\epsilon_{3} \cdot \epsilon_{4}\left(\epsilon_{1} \cdot k_{4} \epsilon_{2} \cdot k_{3}-\epsilon_{1} \cdot k_{3} \epsilon_{2} \cdot k_{4}\right)\right.\right. \\
& +\epsilon_{1} \cdot \epsilon_{2} \epsilon_{3} \cdot \epsilon_{4}+\epsilon_{1} \cdot \epsilon_{2}\left(\epsilon_{3} \cdot k_{2} \epsilon_{4} \cdot k_{1}-\epsilon_{3} \cdot k_{1} \epsilon_{4} \cdot k_{2}\right)
\end{aligned}
$$




$$
\begin{aligned}
& +\frac{1}{2} \epsilon_{1} \cdot \epsilon_{4} \epsilon_{2} \cdot k_{1} \epsilon_{3} \cdot k_{4}+\frac{1}{2} \epsilon_{2} \cdot \epsilon_{3} \epsilon_{1} \cdot k_{2} \epsilon_{4} \cdot k_{3} \\
& \left.-\frac{1}{2} \epsilon_{1} \cdot \epsilon_{3} \epsilon_{2} \cdot k_{1} \epsilon_{4} \cdot k_{3}-\frac{1}{2} \epsilon_{2} \cdot \epsilon_{4} \epsilon_{1} \cdot k_{2} \epsilon_{3} \cdot k_{4}\right] \\
& +\epsilon_{2} \cdot \epsilon_{4}\left(\epsilon_{1} \cdot k_{2} \epsilon_{3} \cdot k_{1}+\epsilon_{1} \cdot k_{3} \epsilon_{3} \cdot k_{4}\right) \\
& +\epsilon_{1} \cdot \epsilon_{4}\left(\epsilon_{2} \cdot k_{4} \epsilon_{3} \cdot k_{1}-\epsilon_{2} \cdot k_{3} \epsilon_{3} \cdot k_{2}\right) \\
& +\epsilon_{2} \cdot \epsilon_{3}\left(\epsilon_{1} \cdot k_{3} \epsilon_{4} \cdot k_{2}-\epsilon_{1} \cdot k_{4} \epsilon_{4} \cdot k_{1}\right) \\
& +\epsilon_{1} \cdot \epsilon_{3}\left(\epsilon_{2} \cdot k_{1} \epsilon_{4} \cdot k_{2}+\epsilon_{2} \cdot k_{4} \epsilon_{4} \cdot k_{3}\right) \\
& -2 \epsilon_{1} \cdot \epsilon_{2} \epsilon_{3} \cdot k_{1} \epsilon_{4} \cdot k_{2}-2 \epsilon_{3} \cdot \epsilon_{4} \epsilon_{1} \cdot k_{3} \epsilon_{2} \cdot k_{4} \\
& +\epsilon_{1} \cdot \epsilon_{4} \epsilon_{2} \cdot \epsilon_{3}+\epsilon_{1} \cdot \epsilon_{3} \epsilon_{2} \cdot \epsilon_{4} \\
& +\left(\epsilon_{1} \cdot k_{4} \epsilon_{2} \cdot k_{3}-\epsilon_{1} \cdot k_{3} \epsilon_{2} \cdot k_{4}\right) \\
& \left.\times\left(\epsilon_{3} \cdot k_{2} \epsilon_{4} \cdot k_{1}-\epsilon_{3} \cdot k_{1} \epsilon_{4} \cdot k_{2}\right)\right\} .
\end{aligned}
$$

By using the above results one proves that the kinematic factor $K\left(k_{i}, \epsilon_{i}\right)$ factorizes into a product of two one-loop kinematic factors. Explicitly we have:

$$
\begin{aligned}
K\left(k_{i}, \epsilon_{i}\right) & \left.\right|_{\left(k_{1}+k_{2}\right)^{2}=-2}=\frac{u^{2}}{4} \epsilon_{1} \cdot \epsilon_{2} \epsilon_{3} \cdot \epsilon_{4}+\frac{u}{2}\left[\epsilon_{1} \cdot \epsilon_{2} \epsilon_{3} \cdot \epsilon_{4}+\epsilon_{1} \cdot \epsilon_{3} \epsilon_{2} \cdot \epsilon_{4}\right. \\
& -\epsilon_{1} \cdot \epsilon_{4} \epsilon_{2} \cdot \epsilon_{3}+\epsilon_{1} \cdot \epsilon_{2}\left(\epsilon_{3} \cdot k_{2} \epsilon_{4} \cdot k_{1}-\epsilon_{3} \cdot k_{1} \epsilon_{4} \cdot k_{2}\right) \\
& +\epsilon_{3} \cdot \epsilon_{4}\left(\epsilon_{1} \cdot k_{4} \epsilon_{2} \cdot k_{3}-\epsilon_{1} \cdot k_{3} \epsilon_{2} \cdot k_{4}\right) \\
& +\epsilon_{1} \cdot \epsilon_{4} \epsilon_{2} \cdot k_{1} \epsilon_{3} \cdot k_{4}+\epsilon_{2} \cdot \epsilon_{3} \epsilon_{1} \cdot k_{2} \epsilon_{4} \cdot k_{3} \\
& \left.-\epsilon_{1} \cdot \epsilon_{3} \epsilon_{2} \cdot k_{1} \epsilon_{4} \cdot k_{3}-\epsilon_{2} \cdot \epsilon_{4} \epsilon_{1} \cdot k_{2} \epsilon_{3} \cdot k_{4}\right] \\
& +\epsilon_{1} \cdot \epsilon_{3}\left(\epsilon_{2} \cdot k_{3} \epsilon_{4} \cdot k_{1}-\epsilon_{2} \cdot k_{1} \epsilon_{4} \cdot k_{3}\right) \\
& +\epsilon_{2} \cdot \epsilon_{4}\left(\epsilon_{1} \cdot k_{4} \epsilon_{3} \cdot k_{2}-\epsilon_{1} \cdot k_{2} \epsilon_{3} \cdot k_{4}\right) \\
& +\epsilon_{2} \cdot \epsilon_{3} \epsilon_{1} \cdot k_{3} \epsilon_{4} \cdot k_{2}+\epsilon_{1} \cdot \epsilon_{4} \epsilon_{2} \cdot k_{4} \epsilon_{3} \cdot k_{1}-\epsilon_{1} \cdot \epsilon_{2} \epsilon_{3} \cdot k_{1} \epsilon_{4} \cdot k_{2} \\
& -\epsilon_{3} \cdot \epsilon_{4} \epsilon_{2} \cdot k_{4} \epsilon_{1} \cdot k_{3}+\epsilon_{1} \cdot \epsilon_{3} \epsilon_{2} \cdot \epsilon_{4} \\
= & \sum_{\alpha} K_{3,1}\left(k_{1}, \epsilon_{1}, k_{2}, \epsilon_{2}, k, \alpha\right) K_{3,1}\left(k_{3}, \epsilon_{3}, k_{4}, \epsilon_{4},-k, \alpha\right) \\
& +\sum_{\sigma} K_{3,2}\left(k_{1}, \epsilon_{1}, k_{2}, \epsilon_{2}, k, \sigma\right) K_{3,2}\left(k_{3}, \epsilon_{3}, k_{4}, \epsilon_{4},-k, \sigma\right) .
\end{aligned}
$$

with $k=-\left(k_{1}+k_{2}\right)$. This completes the study of the factorization of the two-loop four-particle amplitude in superstring theory.

One could use the above result to fix the overall factor of the two-loop four-particle amplitude. In order to do this we need the precise overall factor for the one-loop three-particle amplitude. This factor is unknown in the literature and it should be determined by either using unitarity or factorization at one-loop. We will leave these for a future publication [19].

Note added: Recently the 2-loop 4-particle amplitude in superstring theory was also obtained in [28, 29] and its factorization was studied by D'Hoker, Gutperle and Phong in [30]. 


\section{References}

[1] R. Iengo and Chuan-Jie Zhu, "Two-Loop Computation of the FourParticle Amplitude in Heterotic String Theory," Phys. Lett. 212B (1988) 313-319.

[2] Zhu-Jun Zheng, Jun-Bao Wu and C.-J. Zhu, "Two-Loop Superstrings in Hyperelliptic Language I: the Main Results", Phys. Lett. B559 (2003) 89-98, hep-th/0212191

[3] Zhu-Jun Zheng, Jun-Bao Wu and C.-J. Zhu, "Two-Loop Superstrings in Hyperelliptic Language III: the Four-Particle Amplitude", Nucl. Phys. B663 (2003) 95-122, hep-th/0212219

[4] E. D'Hoker and D.H. Phong, "Two-Loop Superstrings I, Main Formulas", Phys. Lett. B529 (2002) 241-255, hep-th/0110247

[5] E. D'Hoker and D.H. Phong, "Two-Loop Superstrings II, The chiral Measure on Moduli Space", Nucl. Phys. B636 (2002) 3-60, hep-th/0110283

[6] E. D'Hoker and D.H. Phong, "Two-Loop Superstrings III, Slice Independence and Absence of Ambiguities", Nucl. Phys. B636 (2002) 61-79, thp-th/0111016

[7] E. D'Hoker and D.H. Phong, "Two-Loop Superstrings IV, The Cosmological Constant and Modular Forms", Nucl. Phys. B639 (2002) 129-181, hep-th/0111040

[8] For reviews, see E. D' Hoker and D. H. Phong, "Lectures on Two-Loop Superstrings," hep-th/0211111, Lectures delivered at Hangzhou and Beijing, 2002; E. D' Hoker and D. H. Phong, "The Geometry of String Perturbation Theory," Rev. Mod. Phys. 60 (1988) 917-1065.

[9] Eric D' Hoker and D. H. Phong, "Asyzygies, modular forms, and the superstring measure I," hep-th/0411159

[10] Eric D' Hoker and D. H. Phong, "Asyzygies, modular forms, and the superstring measure II," hep-th/0411182

[11] Nathan Berkovits, "Covariant Multiloop Superstring Amplitudes," hep-th/0410079 Strings 2004 talk.

[12] R. Iengo and Chuan-Jie Zhu, "Explicit Modular Invariant TwoLoop Superstring Amplitude Relevant for $R^{4}$," J. High Energy Phys. 9906 (19990 011, hep-th/9905050

[13] R. Iengo, "Computing the $R^{4}$ term at two Superstring Loops," J. High Energy Phys. 0202 (2002) 035, hep-th/0202058

[14] D. J. Gross and E. Witten, "Superstring Modification of Einstein's Equations", Nucl. Phys. B277 (1986) 1.

[15] M. B. Green and M. Gutperle, "Effects of D-instantons", Nucl. Phys. B498 (1997) 195-227, hep-th/9701093

[16] Jun-Bao Wu and Chuan-Jie, "Comments on Two-Loop FourParticle Amplitude in Superstring," J. High Energy Phys. 0305 (2003) 056, hep-th/0303152 
[17] Z. Bern, J. S. Rozowsky and B. Yan, Two-loop four-gluon amplitudes in N = 4 super-Yang-Mills, Phys. Lett. B401 (1997) 273, hep-ph/9702424

[18] Z. Bern, "Perturbative Quantum Gravity and its Relation to Gauge Theory," Living Rev. Rel. 5 (2002) 5, gr-qc/0206071

[19] Zhi-Guang Xiao and Chuan-Jie Zhu, "Factorization and Unitarity in Superstring Theory," hep-th/0503248

[20] Chuan-Jie Zhu, "Factorization of a Two-Loop Four-Particle Amplitude in Superstring Theory," Phys. Lett. 221B (1989) 27-30.

[21] O. Yasuda, "Factorization of a Two-Loop Four-Particle Amplitude in Superstring Theory," Phys. Rev. Lett. 60 (1988) 1688-1691.

[22] M. B. Green and J. H. Schwarz, "Supersymmetric Dual String Theory (III): Loops and Renormalization," Nucl. Phys. B198 (1982) 441-460.

[23] J. Fay, Theta Functions on Riemann Surfaces, Lecture Notes in Mathematics, vol. 352 (Springer-Verlag, Berlin, 1973).

[24] G. Aldazabal, M. Bonini, R. Iengo and C. Nunez, "Superstring Vertex Operators and Scattering Amplitudes on Arbitrary Riemann Surfaces," Nucl. Phys. B307 (1988) 291-318.

[25] I. G. Koh, W. Troost and A. Van Proeyen, Nucl. Phys. B292 (1987) 201-221.

[26] Yoshiaki Tanii and Yoshiyuki Watabiki, "vertex functions in the path integral formalism of string theories,". Nucl. Phys. B316 (1989) 171-196.

[27] Chuan-Jie Zhu, "Superstring Perturbation Theory up to Two Loops and Beyond," SISSA Ph. D. thesis (1989), unpublished.

[28] E. D'Hoker and D. H. Phong, "Two-Loop Superstrings V: Gauge Slice Independence of the N-point Function," hep-th/0501196

[29] E. D'Hoker and D. H. Phong, "Two-Loop Superstrings VI: Non-Renormalization Theorems and the 4-point Function," hep-th/0501197

[30] Eric D' Hoker, M. Gutperle and D. H. Phong, "Two-Loop Superstrings and S-Duality," hep-th/0503180 